\title{
Noise-driven mechanism for pattern formation
}

\author{
J. Buceta, ${ }^{1}$ M. Ibañes, ${ }^{2}$ J. M. Sancho, ${ }^{2}$ and Katja Lindenberg ${ }^{1}$ \\ ${ }^{1}$ Department of Chemistry and Biochemistry, and Institute for Nonlinear Science, University of California, 9500 Gilman Drive, \\ La Jolla, California 92092-0340 \\ ${ }^{2}$ Departament d'Estructura i Constituents de la Matèria, Universitat de Barcelona, Diagonal 647, E-08028 Barcelona, Spain
}

(Received 8 November 2002; published 27 February 2003)

\begin{abstract}
We extend the mechanism for noise-induced phase transitions proposed by Ibañes et al. [Phys. Rev. Lett. 87, 020601 (2001)] to pattern formation phenomena. In contrast with known mechanisms for pure noise-induced pattern formation, this mechanism is not driven by a short-time instability amplified by collective effects. The phenomenon is analyzed by means of a modulated mean field approximation and numerical simulations.
\end{abstract}

DOI: 10.1103/PhysRevE.67.021113 PACS number(s): 05.40.-a, 47.54.+r, 05.10.Gg, 89.75.Kd

\section{INTRODUCTION}

Starting with the seminal work of Horsthemke and Mansour on the Verhulst model [1], noise-induced phenomena have been a subject of intense interest [2]. Much of the early work dealt with noise-induced phenomena in zerodimensional systems. More recently, it has become widely recognized that the effects of fluctuations on systems with a large number of degrees of freedom, so-called spatially extended systems consisting of coupled zero-dimensional systems, can be even more striking [3]. First-order [4,5] and second-order [6-8] noise-induced phase transitions, noiseinduced patterns [9-12], and doubly stochastic resonance [13] are examples that illustrate the broad interest in the subject. The noise intensity is the parameter that controls the creation of order in all these processes. The underlying mechanism that drives the order in these extended systems is a noise-induced short-time instability that is amplified by collective effects which do not occur in the absence of coupling or in the absence of noise. Moreover, as the intensity of the fluctuations increases, a reentrance phenomenon occurs: the noise that first drove the system to an ordered state now restores the disorder. It is important to recall that this particular mechanism is in a sense opposite to that responsible for noise-induced phenomena in zero-dimensional systems $[6,7]$ as these noise-induced phase transitions are observed only when the associated zero-dimensional units exhibit no interesting noise-induced behavior. It was therefore thought for a long time that coupling of zero-dimensional units that undergo a noise-induced transition would exhibit no interesting collective effects.

However, Ibañes et al. [14] introduced a class of exactly solvable models that exhibit both noise-induced transitions in the zero-dimensional case and noise-induced phase transitions in the associated extended system. They stress that this phase transition arises from an effective equilibrium potential in the steady state and does not require a short-time instability or any other reference to the short-time behavior of the system. The key ingredient is the combination of relaxations that rely on field-dependent kinetic coefficients and the disordering effects of external fluctuations. Furthermore, in this case no reentrance phenomenon occurs: the system becomes more ordered with increasing noise intensity.

In this paper, we extend this mechanism to pattern forma- tion. We modify the coupling term of the model in such a way that a morphological instability can appear. We will show, by means of numerical simulations and analytic calculations, that an increasingly ordered spatial structure develops as the intensity of the fluctuation increases beyond a critical value. Moreover, we show that for sufficiently high noise intensity, there is nonmonotonic behavior as a function of the coupling that is, there exists an optimal value of the coupling, for which the ordered structure exhibits maximum coherence.

The paper is organized as follows. We introduce the model in Sec. II and explicitly show that no short-time instability can drive pattern formation in this system. In Sec. III and the Appendix we present a modulated mean-field theory and the phase diagram of the model obtained from this theory. Numerical simulations that confirm the qualitative validity of the theoretical results are presented in Sec. IV. Finally, we summarize our main conclusions in Sec. V

\section{THE MODEL}

As introduced in Ref. [14], we consider the following Langevin dynamics for a space and time dependent scalar field $\phi_{\boldsymbol{r}}(t)$ :

$$
\dot{\phi}_{\boldsymbol{r}}=\Gamma\left(\phi_{\boldsymbol{r}}\right)\left[-a \phi_{\boldsymbol{r}}+\mathcal{L} \phi_{\boldsymbol{r}}\right]+\left[\Gamma\left(\phi_{\boldsymbol{r}}\right)\right]^{1 / 2} \xi(\boldsymbol{r}, t) .
$$

Here, the local force $-a \phi$ arises from a monostable local potential $a \phi^{2} / 2$ and $\mathcal{L}$ stands for the spatial coupling operator. The field-dependent kinetic coefficient is

$$
\Gamma(\phi)=\frac{1}{1+c \phi^{2}} .
$$

The coupling of this kinetic coefficient with the noise favors fluctuations in the disordered state $\phi=0$. Both $a$ and $c$ are positive constants. The noise term is assumed to be Gaussian with zero mean value and correlation function

$$
\left\langle\xi(\boldsymbol{r}, t) \xi\left(\boldsymbol{r}^{\prime}, t^{\prime}\right)\right\rangle=2 \sigma^{2} \delta\left(t-t^{\prime}\right) \delta\left(\boldsymbol{r}-\boldsymbol{r}^{\prime}\right),
$$

where the brackets \langle\rangle denote a statistical average. The associated stochastic integral is interpreted in the Stratonovich sense [2].

In the absence of coupling $(\mathcal{L}=0)$, the model (1) reduces to a collection of zero-dimensional systems that undergo a 
noise-induced transition [2]. At small noise intensity, the stationary probability density has a single maximum at $\phi=0$. As the noise intensity crosses the critical value $\sigma_{c}^{2}=a / c$, the stationary probability density shows two symmetric maxima about a minimum at $\phi=0$. This transition involves no symmetry breaking since in all cases the average $\left\langle\phi_{r}\right\rangle=0$. When sites are coupled diffusively, i.e., $\mathcal{L}=D \nabla^{2}$, the model (1) exhibits a noise-induced phase transition [14]. For a given value of the coupling, as the intensity of the fluctuations increases, the system undergoes a second-order phase transition that involves spontaneous symmetry breaking, that is, a transition from a state with $\left\langle\phi_{r}\right\rangle=0$ to one with $\left\langle\phi_{r}\right\rangle \neq 0$. It is worth noting that when the coupling strength parameter $D$ goes to infinity, the location of that transition point is the same as in a zero-dimensional ensemble, $\sigma_{c}^{2}=a / c$ [14], and that the symmetry breaking in the coupled system can in that case be understood in terms of the dynamics associated with the effective potential in the zero-dimensional case.

We show herein that more complex structural changes can be obtained by modifying the coupling term $\mathcal{L}$. In particular, pattern formation phenomena occur when the system develops a morphological instability, that is, when a Fourier mode of wave vector $\boldsymbol{k}$ other than $\boldsymbol{k}=\mathbf{0}$ becomes unstable [15]. Drawing parallels with previous literature on noise-induced phenomena [9-12], we consider a Swift-Hohenberg coupling, that is [16],

$$
\mathcal{L}=-D\left(k_{0}^{2}+\nabla^{2}\right)^{2} .
$$

The effect of this coupling can be deduced by applying $\mathcal{L}$ to a plane wave $e^{i k \cdot r}$,

$$
\mathcal{L} e^{i k \cdot r}=\omega(k) e^{i k \cdot r},
$$

where $\omega(k)=-D\left(k_{0}^{2}-k^{2}\right)^{2}$ is the (continuous) dispersion relation (we use bold for vectorial quantities and italic for their magnitudes). Thus, the largest eigenvalues, $\omega(k)=0$, are those associated with Fourier modes of wave number magnitude $k=k_{0}$. We will see below that this behavior leads to a morphological instability in the presence of noise. We stress that a morphological instability is a key ingredient of the pattern formation mechanism, but the specific functional form of the coupling term is not.

In the following two sections we show in detail that our model leads to a noise-induced phase transition to patterned states. We end this section by showing explicitly that the transition is not caused by short-time instabilities [9-12]. The relevant short-time evolution equation for the model can be obtained by averaging Eq. (1) and expanding for small $\phi_{r}$ around the average value $\left\langle\phi_{\boldsymbol{r}}\right\rangle=0$ :

$$
\dot{\phi}_{\boldsymbol{r}}=\left(-a-\sigma^{2} c+\mathcal{L}\right) \phi_{\boldsymbol{r}} .
$$

Writing $\phi_{r}(t)$ as a sum (in an infinite system as an integral) over Fourier modes,

$$
\phi_{\boldsymbol{r}}(t)=\sum_{k} \widetilde{\phi}_{\boldsymbol{k}}(t) e^{i \boldsymbol{k} \cdot \boldsymbol{r}}
$$

and integrating, we obtain

$$
\phi_{\boldsymbol{r}}(t)=\sum_{\boldsymbol{k}} \widetilde{\phi}_{\boldsymbol{k}} \exp \left\{\left[-a-\sigma^{2} c+\omega(k)\right] t+i \boldsymbol{k} \cdot \boldsymbol{r}\right\} .
$$

Since $\omega(k) \leqslant 0$, all modes decay and there is no short-time instability.

\section{MODULATED MEAN-FIELD THEORY}

To find the phase diagram for our noisy spatially extended system, we introduce a modulated mean-field theory. In order to implement this theory and, in particular, to calculate the effect of the coupling operator $\mathcal{L}$ in the mean-field context, we need to explicitly distinguish different locations $\boldsymbol{r}$ and $\boldsymbol{r}^{\prime}$ in a way that requires discretization of the system. Since numerical simulations also involve discretization, this procedure does not interfere with the comparisons of theoretical and numerical results. With the understanding of the action of the translation operator

$$
\exp \left(\delta x \frac{\partial}{\partial x}\right) f(x)=f(x+\delta x)
$$

it is straightforward to deduce a discrete version of the SwiftHohenberg coupling operator,

$$
\mathcal{L}=-D\left[k_{0}^{2}+\left(\frac{2}{\Delta x}\right)^{2} \sum_{i=1}^{d} \sinh ^{2}\left(\frac{\Delta x}{2} \frac{\partial}{\partial x_{i}}\right)\right]^{2},
$$

where $d$ stands for the spatial dimension, $\Delta x$ for the lattice spacing, and $\partial / \partial x_{i}$ indicates a partial derivative with respect the $i$ th component of the position vector $r$ $=\left(x_{1}, x_{2}, \ldots, x_{i}, \ldots, x_{d}\right)$. As in the continuous case, the discrete dispersion relation can be obtained by applying the operator (10) to a plane wave $e^{i k \cdot r}$, to obtain

$$
\omega(\boldsymbol{k})=-D\left[k_{0}^{2}-\left(\frac{2}{\Delta x}\right)^{2} \sum_{i=1}^{d} \sin ^{2}\left(\frac{\Delta x}{2} k_{i}\right)\right]^{2} .
$$

Here, $k_{i}$ denotes component $i$ of the wave vector $\boldsymbol{k}$ $=\left(k_{1}, k_{2}, \ldots, k_{i}, \ldots, k_{d}\right)$.

Note that as in the continuous problem, $\omega(\boldsymbol{k})$ is nonpositive for any value of $k$, but that in the discrete case it depends not only on the magnitude but also on the direction of $\boldsymbol{k}$. Of particular importance in our subsequent analysis are those modes for which $\omega(\boldsymbol{k})=0$. In the continuum, these are the modes with $k=k_{0}$, which are all those that lie on a continuous hypersurface in reciprocal space of radius $k_{0}$ around the origin. In the discretized system the magnitudes $k^{*}$ of the most unstable modes are shifted from $k_{0}$ and depend on direction, as can be seen by solving Eq. (11). The longest vectors such that $\omega\left(\boldsymbol{k}^{*}\right)=0$ lie along the Cartesian directions, e.g. $\left(k^{*}, 0,0, \ldots, 0\right)$ and have magnitude

$$
\max k^{*}=\frac{2}{\Delta x} \arcsin \left(\frac{k_{0} \Delta x}{2}\right) .
$$

The shortest lie along a reciprocal space diagonal, e.g., $\left(k^{*}, k^{*}, k^{*}, \ldots, k^{*}\right) / \sqrt{d}$, and have magnitude 


$$
\min k^{*}=\frac{2 \sqrt{d}}{\Delta x} \arcsin \left(\frac{k_{0} \Delta x}{2 \sqrt{d}}\right) .
$$

If $k_{0} \Delta x \leqslant 1$ (which it always will be in our analysis), then the difference between these two values is smaller than $3 \%$. It, is therefore, only a mild approximation to neglect the directional dependence of the solutions of $\omega\left(\boldsymbol{k}^{*}\right)=0$ and focus on the magnitude, $\omega\left(k^{*}\right)=0$.

To establish the existence of patterns of a characteristic length scale, we seek a spatially periodic structure defined by wave vectors $\boldsymbol{k}$, whose magnitude $k$, is associated with the inverse of this length scale. As we shall see, the appropriate wave vectors to focus on are precisely those of magnitude $k^{*}$, that is, those for which $\omega(\boldsymbol{k})=0$. As is customary in mean field theories, we make an ansatz about the behavior of the field at sites $\boldsymbol{r}^{\prime}$ other than the focus point $\boldsymbol{r}$ which are coupled to it by the operator $\mathcal{L}$, one that incorporates an appropriate spatial modulation:

$$
\phi_{\boldsymbol{r}^{\prime}}=\mathcal{A}\left(k^{*}\right) \sum_{\left\{\boldsymbol{k}^{*}\right\}} \cos \left[\boldsymbol{k} \cdot\left(\boldsymbol{r}-\boldsymbol{r}^{\prime}\right)\right],
$$

where the sum (or, in an infinite system, the integral) is over wave vectors of magnitude $k^{*}$. Our ansatz thus also incorporates the assumption that all modes of this magnitude contribute with equal (direction-independent) weight $\mathcal{A}\left(k^{*}\right)$. In the Appendix, we present details of some of the steps that show the action of the coupling operator on this ansatz, to arrive at the result

$$
\mathcal{L} \phi_{\boldsymbol{r}}=D_{1}\left[\mathfrak{n}\left(k^{*}\right) \mathcal{A}\left(k^{*}\right)-\phi_{\boldsymbol{r}}\right]
$$

where

$$
\mathfrak{n}\left(k^{*}\right)=\frac{d \pi^{d / 2}}{\Gamma(d / 2+1)}\left(\frac{N k^{*}}{2 \pi}\right)^{d-1}
$$

is the number of modes of magnitude $k^{*}$, and

$$
D_{1}=D\left[\left(\frac{2 d}{(\Delta x)^{2}}-k_{0}^{2}\right)^{2}+\frac{2 d}{(\Delta x)^{4}}\right]
$$

Substitution in Eq. (1) then leads to an equation that depends only on a generic site index $\boldsymbol{r}$ that can simply be dropped:

$$
\begin{aligned}
\dot{\phi}= & \Gamma(\phi)\left\{-a \phi+D_{1}\left(k^{*}\right)\left[\mathfrak{n}\left(k^{*}\right) \mathcal{A}\left(k^{*}\right)-\phi\right]\right\} \\
& +[\Gamma(\phi)]^{1 / 2} \xi(t),
\end{aligned}
$$

and $\xi(t)$ is zero-centered Gaussian noise $\delta$-correlated in time,

$$
\left\langle\xi(t) \xi\left(t^{\prime}\right)\right\rangle=\frac{2 \sigma^{2}}{(\Delta x)^{d}} \delta\left(t-t^{\prime}\right)
$$

Here, we have incorporated the fact that the continuum delta function $\delta\left(\boldsymbol{r}-\boldsymbol{r}^{\prime}\right)$ has been replaced in the usual way by a ratio that contains the Kronecker delta and the lattice spacing, $\delta_{r, r^{\prime}} /(\Delta x)^{2}$. Henceforth, we set $\Delta x=1$.
The amplitude $\mathcal{A}\left(k^{*}\right)$ is the mean field quantity that must be chosen self-consistently to complete the solution of the problem. The stationary probability density for the stochastic process (15) is

$$
\begin{aligned}
\rho\left(\phi ; \mathcal{A}\left(k^{*}\right)\right) \\
=\mathcal{N}\left[\mathfrak{n}\left(k^{*}\right) \mathcal{A}\left(k^{*}\right)\right]\left(1+c \phi^{2}\right)^{1 / 2} \\
\quad \times \exp \left\{-\frac{1}{\sigma^{2}}\left[\frac{1}{2}\left(a+D_{1}\right) \phi^{2}-D_{1} \mathfrak{n}\left(k^{*}\right) \mathcal{A}\left(k^{*}\right) \phi\right]\right\},
\end{aligned}
$$

where the normalization constant $\mathcal{N}\left[\mathfrak{n}\left(k^{*}\right) \mathcal{A}\left(k^{*}\right)\right]$ depends on the amplitude and must therefore be carefully included in the self-consistent solution. Self-consistency is then embodied in the assumption that $\mathfrak{n}\left(k^{*}\right) \mathcal{A}\left(k^{*}\right)$ is the average value of the field at any point in space, i.e., in the requirement that

$$
\mathfrak{n}\left(k^{*}\right) \mathcal{A}\left(k^{*}\right)=\int_{-\infty}^{\infty} \phi \rho\left(\phi ; \mathcal{A}\left(k^{*}\right)\right) d \phi
$$

which is appropriate either if $\mathcal{A}\left(k^{*}\right)=0$ and the distribution is symmetric in $\phi$, or if $\mathfrak{n}\left(k^{*}\right) \mathcal{A}\left(k^{*}\right)$ is much larger than the (appropriately phased) combined amplitudes of all the other modes. The latter occurs if there is an instability that leads to the formation of a pattern with wave numbers of magnitude $k^{*}$.

Since $\rho(\phi ; 0)=\rho(-\phi ; 0)$, it follows that $\mathcal{A}\left(k^{*}\right)=0$ is always a solution. To find other solutions, we expand the integral on the right side of Eq. (21) around $\mathcal{A}\left(k^{*}\right)=0$,

$$
\int_{-\infty}^{\infty} \phi \rho\left(\phi ; \mathcal{A}\left(k^{*}\right)\right) d \phi=b \mathcal{A}\left(k^{*}\right)+O\left(\mathcal{A}^{3}\left(k^{*}\right)\right)
$$

where

$$
b=\left.\int_{-\infty}^{\infty} \phi \frac{\partial \rho\left(\phi ; \mathcal{A}\left(k^{*}\right)\right)}{\partial \mathcal{A}\left(k^{*}\right)}\right|_{\mathcal{A}\left(k^{*}\right)=0} d \phi .
$$

It follows that self-consistent solutions different from $\mathcal{A}\left(k^{*}\right)=0$ are possible, and that the loci that indicate the appearance of these solutions satisfy $b=\mathfrak{n}\left(k^{*}\right)$, that is,

$$
\frac{D_{1}}{\sigma^{2}} \int_{-\infty}^{\infty} \phi^{2} \rho(\phi ; 0) d \phi=1 .
$$

The latter condition, which determines the phase diagram of the model, can also be obtained by geometrical arguments $[3,17]$. Equation $(24)$ can be expressed in the following algebraic form: 


$$
1=\frac{D_{1} \mathbb{K}_{1}\left(\frac{a+D_{1}}{4 c \sigma^{2}}\right) e^{\left(a+D_{1}\right) / 4 c \sigma^{2}}}{2 \sigma^{2} c \sqrt{\pi} \mathrm{U}\left(-\frac{1}{2}, 0, \frac{a+D_{1}}{2 c \sigma^{2}}\right)},
$$

where $\mathrm{U}(x, y, z)$ is the confluent hypergeometric function and $\mathbb{K}_{n}(x)$ is the Bessel function of the second kind.

The derivation is not complete until we confirm, at least within the approximations implemented in our mean field approach, that the most unstable modes are indeed those of magnitude $k^{*}$, i.e., those for which $\omega(\boldsymbol{k})=0$. This is easily ascertained as follows. If we consider an ansatz state of the form (14) but with a different $k$, then we would arrive at a mean field equation containing $\omega(k)$ explicitly:

$$
\begin{aligned}
\dot{\phi}= & \Gamma(\phi)\left\{-(a-\omega(k)) \phi+D_{e f f}(k)[\mathfrak{n}(k) \mathcal{A}(k)-\phi]\right\} \\
& +[\Gamma(\phi)]^{1 / 2} \xi(t),
\end{aligned}
$$

where $D_{\text {eff }}(k)=D_{1}+\omega(k)$ [cf. Eq. (A7)]; here the assumption has again been made that $\omega(\boldsymbol{k})$ is well approximated by $\omega(k)$. Note that if $\omega(k)=0$ we recover Eq. (18). If we now follow the same steps leading to the condition (25) which marks the boundary of pattern formation, we find that the only modification is that the very first $D_{1}$ in the numerator is now replaced with $D_{e f f}(k)$. The other occurrences of $D_{1}$ are not affected because $D_{1}$ and $a$ are each shifted in such a way that their sum remains unchanged. Since $D_{\text {eff }}(k)<D_{1}$ for all $k \neq k^{*}$, it follows that in order to satisfy Eq.(25) the noise intensity has to be greater for other $k$. In other words, while modes other than those of magnitude $k^{*}$ may become unstable, they first do so at higher values of the noise intensity. Conversely, for a given noise intensity, the coupling must be stronger to produce an instability of other wave vectors. Thus, as the noise intensity is increased from zero for a given coupling, or the coupling is increased from zero for a given noise intensity beyond $\sigma_{c}^{2}$, the first and hence strongest instability occurs at $k^{*}$.

Figure 1 shows the phase diagram of the model in the space $\left(\sigma^{2}, D\right)$ obtained from the numerical solution of Eq. (25) in two dimensions with $\Delta x=1, a=1, c=3$, and $k_{0}$ $=1$, which leads to $k^{*} \approx 1.035$. Note that as $D$ goes to infinity, the transition line moves to the value of the noise intensity where the zero-dimensional stationary probability density becomes bimodal, $\sigma_{c}^{2}=1 / 3$.

Since Eq. (1) satisfies the inversion symmetry $\phi \leftrightarrow-\phi$, roll-shaped patterns are likely [15]. In order to characterize the structure that develops, we use as an order parameter the total power spectrum at the most unstable modes,

$$
S\left(k^{*}\right)=\sum_{\left\{\boldsymbol{k}^{*}\right\}} \widetilde{\phi}_{\boldsymbol{k}^{*}} \widetilde{\phi}_{-\boldsymbol{k}^{*}}
$$

where, as before, $\widetilde{\phi}_{\boldsymbol{k}}$ stands for the Fourier transform of the field $\phi_{\boldsymbol{r}}$ and the sum is over all modes of magnitude $k^{*}$. This parameter characterizes the transition from a laminar regime

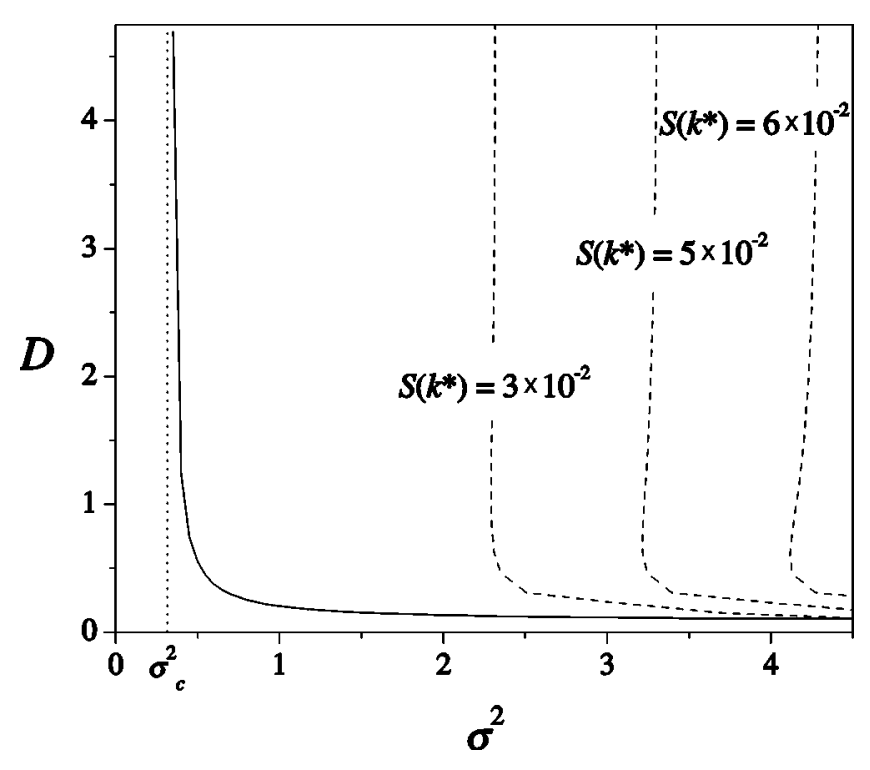

FIG. 1. Phase diagram of the model obtained from the modulated mean field theory. The wide solid line indicates the transition loci $(25)$ in $\left(D, \sigma^{2}\right)$ space for $k_{0}=1\left(k^{*} \approx 1.035\right), a=1$, and $c$ $=3$. Inside the ordered region where patterns develop we have indicated some contour lines labeled by the value of the order parameter $S\left(k^{*}\right)$ (see text). Note that as the coupling goes to infinity, the transition line tends to the value of the noise intensity where the zero-dimensional system undergoes a noise-induced transition, $\sigma_{c}^{2}$ $=1 / 3$.

(homogeneous) to a convective regime (roll-like patterns) that we expect to obtain here. Using our self-consistent solution then gives, in the modified mean-field approximation, the appropriately normalized relation

$$
S\left(k^{*}\right)=\mathfrak{n}\left(k^{*}\right) \mathcal{A}^{2}\left(k^{*}\right)
$$

In Fig. 1, we also present several contour lines indicating the value of $S\left(k^{*}\right)$. Note that for a given value of the coupling $D$, as noise intensity increases, the order parameter $S\left(k^{*}\right)$ also increases, that is, the stronger the noise, the larger is the amplitude of structures associated with wave vector magnitude $k^{*}$. Note also that for sufficiently large noise intensity, for a given value of $\sigma^{2}$ there is a nonmonotonic behavior of $S\left(k^{*}\right)$ as a function of the coupling strength, indicating that there exists a value of the coupling for which the structures associated with $k^{*}$ exhibit maximum coherence. At the same time, the possible instability of other modes near $k^{*}$ may affect the actual physical appearance of the system, so that these effects may not be visually unequivocal.

Finally, we note that it is common in pattern formation discussions to use the so-called flux of convective heat

$$
J=\frac{1}{N^{d}} \sum_{r} \phi_{r}^{2}=\sum_{k} \widetilde{\phi}_{\boldsymbol{k}} \widetilde{\phi}_{-\boldsymbol{k}}
$$

as an order parameter. If our ansatz state were exact, then in 


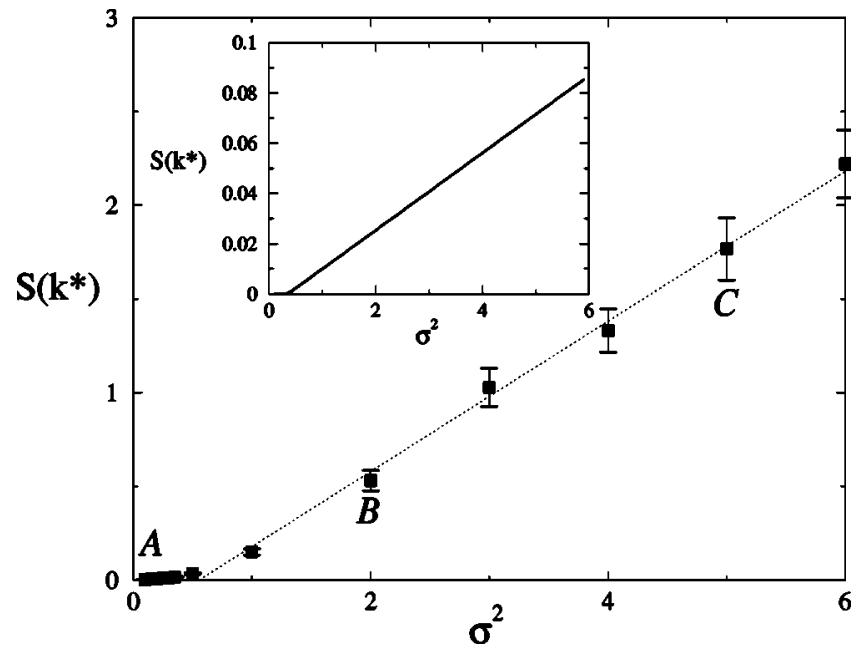

FIG. 2. Order parameter as a function of the noise intensity obtained from numerical simulations (squares) for $a=1, c=3, D$ $=5$, and $k_{0}=1$. The error bars are indicated. The dashed line indicates the linear interpolation of the numerical data used to locate the transition point, $\sigma_{0}^{2}=0.5 \pm 0.1$. The points labeled $A\left(\sigma^{2}=0.1\right), B$ $\left(\sigma^{2}=2\right)$, and $C\left(\sigma^{2}=5\right)$ correspond to the spatial structures shown in Fig. 3. Inset: mean-field result, with transition point at $\sigma_{0}^{2}$ $=0.34955 \pm 10^{-5}$.

the patterned state the two would be identical, $J=S\left(k^{*}\right)$. Differences point to the presence of other unstable modes.

\section{NUMERICAL SIMULATIONS}

In order to check the analytical predictions of the modified field theory, we perform numerical simulations of Eq. (1) on a two-dimensional square lattice of $64 \times 64$ cells and apply Dirichlet-Neumann boundary conditions commonly used in studies of fluids, namely, the field and the normal component of the gradient are zero at the boundaries. The relevant parameters have the following values in our simulations: mesh size $\Delta x=1$ that gives the system length scale $L=N \Delta x=64$, time step $\Delta t=0.001, k_{0}=1$, which leads to $k^{*} \approx 1.035$ and an aspect ratio $\Lambda=k^{*} L / 2 \pi \sim 10$. The other parameters of the model are again taken as $a=1$ and $c=3$. Using these values, the onset of bistability of the stationary one-site potential occurs at $\sigma_{c}^{2}=a / c=1 / 3$. We expect the transition to pattern formation to occur for noise intensities near $\sigma_{c}^{2}$ if the coupling is sufficiently strong. According to the mean field phase diagram Fig. $1, D=5$ is sufficiently large. We use this value of the coupling in subsequent calculations and figures. Although the initial conditions do not matter for the final outcome, the outcome is reached more quickly if we start from a targetlike pattern of rings of width $k^{*}$. We have ascertained that other initial conditions, for example, a random initial condition, reach the stationary state albeit in a longer time.

We have numerically computed the order parameter for several noise intensities, and the results are shown in Fig. 2. The order parameter is very small for low noise intensities until the critical noise intensity is reached, after which the order parameter grows linearly with $\sigma^{2}$. Our simulations
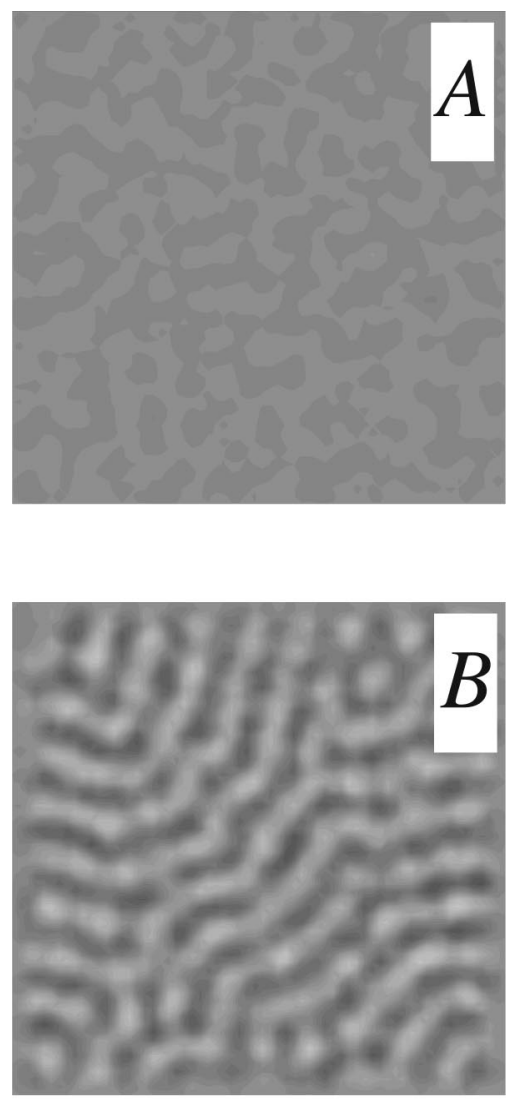

$\phi=-6$
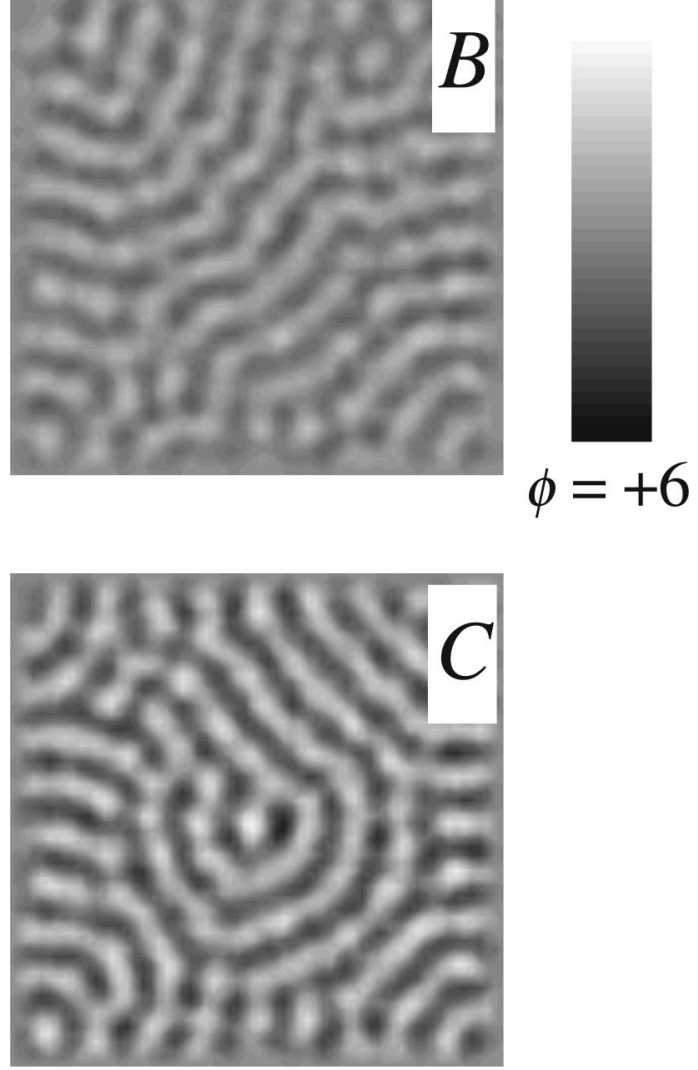

FIG. 3. Density plots of the stationary field associated with the points $A$ (first panel), $B$ (second), and $C$ (third) of Fig. 2. Note that as predicted, for a constant value of the coupling, a pattern develops as the intensity of the noise increases.

lead to a critical value $\sigma_{0}^{2}=0.5 \pm 0.1$. The mean field result, also shown in the figure, predicts the ordering transition to occur at $\sigma_{0}^{2}=0.34955 \pm 10^{-5}$ and also captures the linear transcritical behavior of $S\left(k^{*}\right)$ with $\sigma^{2}$, but predicts a slower growth of the order parameter with noise intensity than the numerical simulations. Nevertheless, the mean-field theory clearly captures the full qualitative behavior of the system, especially near the transition point.

We have also computed the flux according to Eq. (29) for all the cases for which we have presented the order param- 


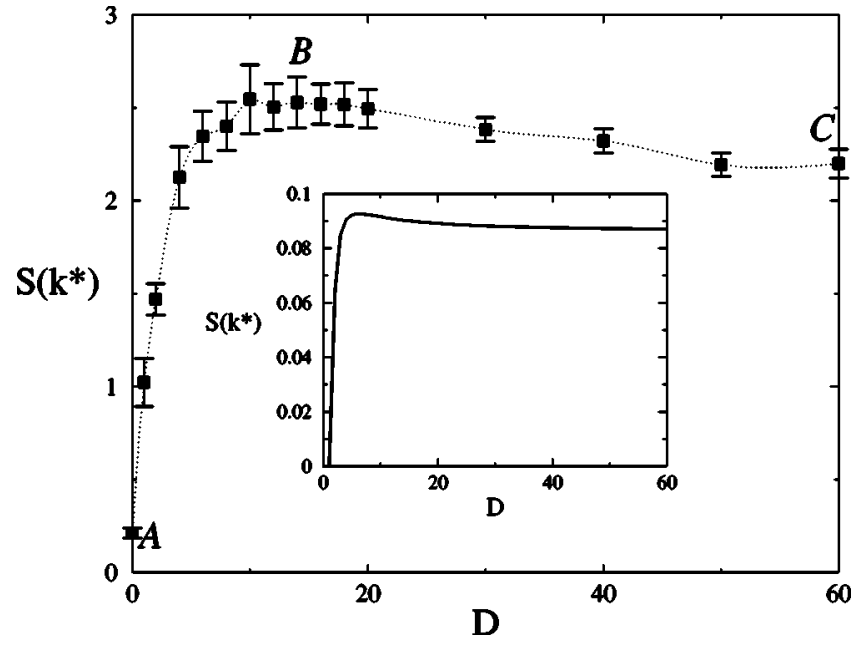

FIG. 4. Order parameter as a function of the coupling strength obtained from numerical simulations (squares) for $a=1, c=3, \sigma^{2}$ $=6$, and $k_{0}=1$. The error bars are indicated; the dashed line is a guide for the eye. The points labeled $A$ ( $D=0$, uncoupled system), $B(D=14)$, and $C(D=60)$ correspond to the spatial structures shown in Fig. 5. Inset: mean-field result.

eter. We find that both predict essentially the same critical noise value for the appearance of patterns, and that for our simulation parameters, the flux is fairly consistently twice as large as the order parameter (because of the contributions of other modes to the former). This consistency would indicate that even well beyond the transition point, the most unstable modes dominate the flux.

The spatial pattern that emerges as $\sigma^{2}$ increases is illustrated in Fig. 3 using density plots of the stationary value of the field for the noise intensities labeled $A, B$, and $C$ in Fig. 2: $\sigma_{A}^{2}=0.1$ (weak noise, no pattern), $\sigma_{B}^{2}=2$ (rolls are visible), and $\sigma_{C}^{2}=5$ (strong noise, distinctive pattern).

In order to ascertain the nonmonotonic behavior of the order parameter with coupling strength at a given noise intensity, in Fig. 4 we present the results for a fixed noise intensity $\sigma^{2}=6$. Simulations are indicated by squares, and the inset shows the results predicted by the theory. While there are again quantitative differences, the qualitative agreement is evident: both curves show a clear nonmonotonic behavior. The density plots of the field associated with points $A, B$, and $C$ in Fig. 5 confirm this behavior. Of course even as the amplitudes of wave vectors of magnitude $k^{*}$ are decreasing, the amplitudes of other nearby wave vectors may be growing. Therefore, while the lightening of pattern $C$ on the gray scale reflects the decrease in the order parameter, a visual perception of some loss of distinctness could be due to an admixture of other wave vectors.

\section{CONCLUSIONS}

We have shown by means of a modulated mean-field approximation and numerical simulations that the mechanism for noise-induced phase transitions introduced by Ibañes et al. [14] can be extended to pattern formation phenomena. In contrast with previous work on noise-induced patterns, no
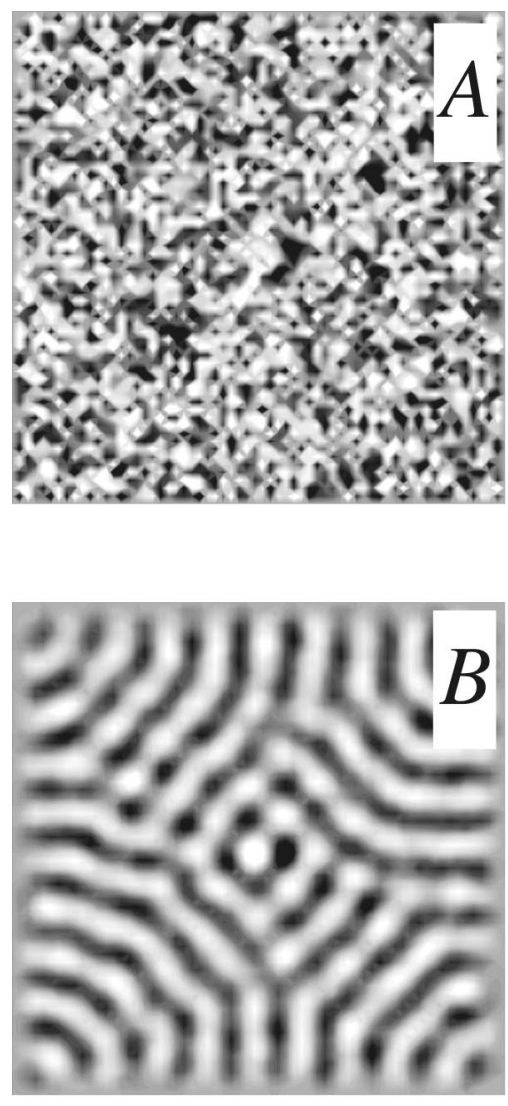

$\phi=-4$
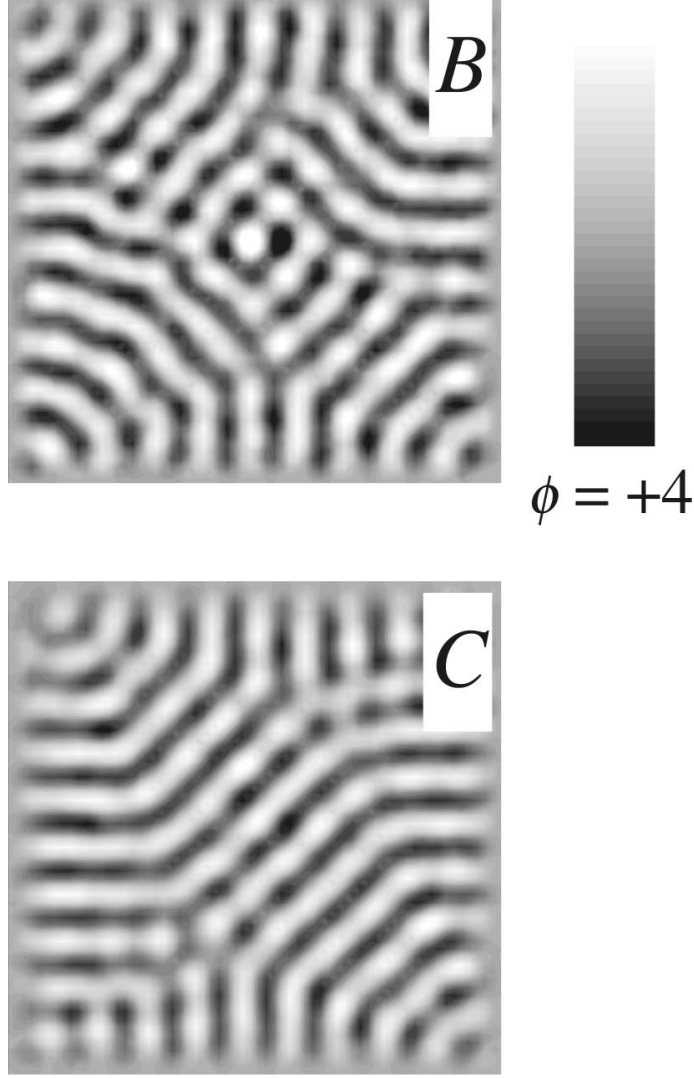

FIG. 5. Density plots of the stationary field associated with the points $A$ (first panel), $B$ (second), and $C$ (third) of Fig. 4. Note that, as predicted, for a constant value of the noise, a pattern develops as the coupling increases but then becomes less distinct as the coupling increases further.

short-time instability is required to generate these spatial structures. As a consequence, this transition is independent of the noise interpretation, as has been shown for noiseinduced phase transitions [18]. For example, for the Ito interpretation we have checked numerically that this model exhibits the same noise-induced patterns, but at a lower critical noise intensity. Furthermore, no reentrance phenomenon occurs as the fluctuations grow in intensity. Indeed, in our system, stronger noise leads to increasingly ordered struc- 
tures. The mean-field theory has allowed us to characterize the model by computing its phase diagram. We have also performed numerical simulations that confirm the qualitative validity of the theoretical analysis. We find that as the intensity of the fluctuations increases, a rotationally symmetric roll-shaped pattern appears. The pattern is characterized by the most unstable modes of the system, those with wave vector of a magnitude $k=k^{*}$ that is explicitly predicted by the mean field analysis. Both the theoretical and numerical analyses show that as the coupling between sites goes to infinity, the transition to pattern formation occurs at the same point where the zero-dimensional system presents a noiseinduced transition. We have also shown that for sufficiently strong noise intensity, the order parameter for the system is nonmonotonic as a function of the coupling strength. Thus, for sufficiently strong noise, there exists an optimal value of the coupling such that the patterns of characteristic size $2 \pi / k^{*}$ are maximally coherent.

\section{ACKNOWLEDGMENTS}

This work was partially supported by the National Science Foundation under Grant No. PHY-9970699, by MECDSpain Grant No. EX2001-02880680, and by MCYT-Spain Grant Nos. BFM2001-0291 and BFM2000-0624.

\section{APPENDIX: IMPLEMENTATION OF THE MODIFIED MEAN-FIELD THEORY}

We begin by exhibiting in some detail the dependences associated with the ansatz field (14) and the action of $\mathcal{L}$ on it. For example, for an $\boldsymbol{r}^{\prime}$ that is $m$ lattice sites away from $\boldsymbol{r}$ $=\left(x_{1}, x_{2}, \ldots, x_{d}\right)$ in the direction $j$, the ansatz reads

$$
\phi\left(x_{1}, x_{2}, \ldots, x_{j}+m \Delta x, \ldots, x_{d}\right)=\sum_{\left\{k^{*}\right\}} \mathcal{A}\left(k^{*}\right) \cos \left(m \Delta x k_{j}\right) .
$$

For an $\boldsymbol{r}^{\prime}$ that is in the immediate positive diagonal location away from $\boldsymbol{r}$ we have

$$
\begin{gathered}
\phi\left(x_{1}+\Delta x, x_{2}+\Delta x, \ldots, x_{j}+\Delta x, \ldots, x_{d}+\Delta x\right) \\
=\sum_{\left\{k^{*}\right\}} \mathcal{A}\left(k^{*}\right) \cos \left[\Delta x\left(k_{1}+k_{2}+\cdots+k_{d}\right)\right] .
\end{gathered}
$$

Next, to apply the discrete version (10) of $\mathcal{L}$ we must elucidate the effect of the operators $\left[\sum_{i=1}^{d} \sinh ^{2}\left((\Delta x / 2)\left(\partial / \partial x_{i}\right)\right)\right]^{n}$ on the field $\phi_{r}$ for $n=1,2$. With $n=1$, we use the relation $2 \sinh ^{2}(y / 2)=[\cosh (y)-1]$ and note that

$$
\begin{aligned}
\sum_{i=1}^{d} \cosh \left(\Delta x \frac{\partial}{\partial x_{i}}\right) \phi_{r} \\
=\frac{1}{2}\left[\phi\left(x_{1}+\Delta x, x_{2}, \ldots, x_{j}, \ldots, x_{d}\right)\right. \\
\quad+\phi\left(x_{1}-\Delta x, x_{2}, \ldots, x_{j}, \ldots, x_{d}\right)+\ldots \\
\quad+\phi\left(x_{1}, x_{2}, \ldots, x_{j}+\Delta x, \ldots, x_{d}\right) \\
\quad+\phi\left(x_{1}, x_{2}, \ldots, x_{j}-\Delta x, \ldots, x_{d}\right)+\ldots \\
\quad+\phi\left(x_{1}, x_{2}, \ldots, x_{j}, \ldots, x_{d}+\Delta x\right) \\
\left.\quad+\phi\left(x_{1}, x_{2}, \ldots, x_{j}, \ldots, x_{d}-\Delta x\right)\right] .
\end{aligned}
$$

By using Eq. (A1) in this last equation, we obtain

$$
\sum_{i=1}^{d} \cosh \left(\Delta x \frac{\partial}{\partial x_{i}}\right) \phi_{\boldsymbol{r}}=\sum_{\left\{k^{*}\right\}} \mathcal{A}\left(k^{*}\right) \sum_{i=1}^{d} \cos \left(k_{i} \Delta x\right) .
$$

As for $n=2$, we note that $4 \sinh ^{2}(y / 2) \sinh ^{2}(z / 2)=[\cosh (y)$ $-1][\cosh (z)-1]$ and, in turn, $\cosh (y) \cosh (z)=\frac{1}{2}[\cosh (y+z)$ $+\cosh (y-z)]$. The latter combination leads to contributions that involve both forward and backward translations in different spatial directions. This is easily visualized by noting explicitly that

$$
\begin{aligned}
{\left[\sum_{i=1}^{d} \cosh \left(\Delta x \frac{\partial}{\partial x_{i}}\right)\right]^{2}=} & \frac{1}{2}\left\{\sum_{i, j=1}^{d} \cosh \left[\Delta x\left(\frac{\partial}{\partial x_{i}}+\frac{\partial}{\partial x_{j}}\right)\right]\right. \\
& \left.+\cosh \left[\Delta x\left(\frac{\partial}{\partial x_{i}}-\frac{\partial}{\partial x_{j}}\right)\right]\right\} .
\end{aligned}
$$

Notice that for the $d$ cases where $i=j$, the second term on the right hand side leaves the field at the original site $r$. The field at the original site is not represented by the ansatz assumption, and therefore we must subtract the $d$ "spurious" terms produced by the ansatz state and add $d$ times the field $\phi_{r}$. This procedure leads to

$$
\begin{aligned}
{\left[\sum_{i=1}^{d} \cosh \left(\Delta x \frac{\partial}{\partial x_{i}}\right)\right]^{2} \phi_{r} } & =\frac{d}{2} \phi_{r}+\sum_{\left\{k^{*}\right\}} \mathcal{A}\left(k^{*}\right) \\
& \times\left[\left(\sum_{i=1}^{d} \cos \left(k_{i} \Delta x\right)\right)^{2}-\frac{d}{2}\right] .
\end{aligned}
$$

Note that we have taken advantage of the directional insensitivity of $k^{*}$.

Use of Eqs. (A4) and (A6) in Eq. (10) then leads to the following approximation for the term containing the SwiftHohenberg coupling operator: 


$$
\begin{aligned}
\mathcal{L} \phi_{\boldsymbol{r}} & =D_{1}\left(\sum_{\left\{k^{*}\right\}} \mathcal{A}\left(k^{*}\right)-\phi_{\boldsymbol{r}}\right)+\sum_{\left\{k^{*}\right\}} \mathcal{A}\left(k^{*}\right) \omega\left(k^{*}\right) \\
& =D_{1}\left(\sum_{\left\{k^{*}\right\}} \mathcal{A}\left(k^{*}\right)-\phi_{\boldsymbol{r}}\right)
\end{aligned}
$$

where the last term in the first line vanishes because $\omega\left(k^{*}\right)$ $=0$, and where

$$
D_{1}=D\left[\left(\frac{2 d}{\Delta x^{2}}-k_{0}^{2}\right)^{2}+\frac{2 d}{\Delta x^{4}}\right] .
$$

Finally, since the summand in Eq. (A7) is independent of the direction of the $\boldsymbol{k}^{*}$, the sums simply give the number of terms in the sum (or the appropriate integral form) times the summand. Simulations always involve a finite system of $N^{d}$ sites i.e., of volume $(N \Delta x)^{d}$, so that the allowed modes themselves form a discrete set, with each component separated from the next one by an interval $\Delta k=2 \pi / N \Delta x$. One way to count the number of modes $\mathfrak{n}\left(k^{*}\right)$ in the sum is to construct a ring of radius min $k^{*}$ [which we shall simply call $k^{*}$ following the discussion surrounding Eqs. (12) and (13)] of thickness $\Delta k \equiv 2 \pi / N \Delta x$, and to consider all the modes that lie in this ring. We can then estimate this number by calculating the number of cells of volume $(2 \pi / N \Delta x)^{d}$ in the ring:

$$
\mathfrak{n}\left(k^{*}\right)=\frac{d \pi^{d / 2}}{\Gamma(d / 2+1)}\left(\frac{N k^{*}}{2 \pi}\right)^{d-1}
$$

Although variations in the particular way of counting are possible, for sufficiently large $N$ the differences are small. Thus, we finally arrive at the mean-field approximation

$$
\mathcal{L} \phi_{\boldsymbol{r}}=D_{1}\left[\mathfrak{n}\left(k^{*}\right) \mathcal{A}\left(k^{*}\right)-\phi_{\boldsymbol{r}}\right]
$$

[1] W. Horsthemke and M. Malek Mansour, Z. Phys. B 24, 307 (1976).

[2] W. Horsthemke and R. Lefever, Noise-Induced Transitions (Springer, Berlin, 1984).

[3] J. García-Ojalvo and J.M. Sancho, Noise in Spatially Extended Systems (Springer, New York, 1999).

[4] R. Müller, K. Lippert, A. Kühnel, and U. Behn, Phys. Rev. E 56, 2658 (1997).

[5] O. Carrillo, M. Ibañes, and J.M. Sancho, Fluct. Noise Lett. 2, L1 (2002).

[6] C. Van den Broeck, J.M.R. Parrondo, and R. Toral, Phys. Rev. Lett. 73, 3395 (1994).

[7] C. Van den Broeck, J.M.R. Parrondo, R. Toral, and R. Kawai, Phys. Rev. E 55, 4084 (1997).

[8] J. García-Ojalvo, J.M.R. Parrondo, J.M. Sancho, and C. Van den Broeck, Phys. Rev. E 54, 6918 (1996).

[9] J. García-Ojalvo, A. Hernández-Machado, and J.M. Sancho, Phys. Rev. Lett. 71, 1542 (1993).
[10] A. Becker and L. Kramer, Phys. Rev. Lett. 73, 955 (1994); A. Becker and L. Kramer, Physica D 90, 408 (1995).

[11] J.M.R. Parrondo, C. Van den Broeck, J. Buceta, and F.J. de la Rubia, Physica A 224, 153 (1996).

[12] A.A. Zaikin and L. Schimansky-Geier, Phys. Rev. E 58, 4355 (1998).

[13] A.A. Zaikin, J. Kurths, and L. Schimansky-Geier, Phys. Rev. Lett. 85, 227 (2000).

[14] M. Ibañes, J. García-Ojalvo, R. Toral, and J.M. Sancho, Phys. Rev. Lett. 87, 020601 (2001).

[15] M.C. Cross and P.C. Hohenberg, Rev. Mod. Phys. 65, 851 (1993).

[16] J. Swift and P.C. Hohenberg, Phys. Rev. A 15, 319 (1977).

[17] C. Van den Broeck, J.M.R. Parrondo, J. Armero, and A. Hernández-Machado, Phys. Rev. E 49, 2639 (1994).

[18] O. Carrillo, M. Ibañes, J. García-Ojalvo, J. Casademunt, and J.M. Sancho (unpublished). 